\title{
Production of a Fully Spin-Polarized Ensemble of Positronium Atoms
}

\author{
D. B. Cassidy, V.E. Meligne, and A. P. Mills, Jr. \\ Department of Physics and Astronomy, University of California, Riverside, California 92521-0413, USA
}

(Received 15 February 2010; published 27 April 2010)

\begin{abstract}
Long-lived $|m|=1$ positronium (Ps) atoms are produced in vacuum when high density bursts of positrons with net polarization $p_{0}$ are implanted into a porous silica film in a $2.3 \mathrm{~T}$ magnetic field. We observe a decrease in the $|m|=1$ population as the density of the incident positron beam is increased due to quenching interactions between oppositely polarized Ps atoms within the target. Saturation of this density dependent quenching indicates that the initial positron spin polarization $p_{0}=28 \pm 1 \%$, and demonstrates the long term $\left(10^{2} \mathrm{~s}\right)$ survival of positron polarization in a Surko-type buffer gas trap. We conclude that, at high Ps densities, the minority spin component is essentially eliminated and the remaining Ps is almost entirely ( $\sim 96 \%)$ polarized, as required for the formation of a Ps Bose-Einstein condensate.
\end{abstract}

DOI: 10.1103/PhysRevLett.104.173401

PACS numbers: $36.10 . \mathrm{Dr}, 34.80 . \mathrm{Nz}$

As a result of parity nonconservation in the weak interactions [1], $\beta^{ \pm}$particles emitted with velocity $v$ from a radioactive nucleus have helicity $h= \pm v / c$ [2]. This gift of nature means that slow positron beams derived from radioactive sources [3] are always spin polarized to some extent, because the asymmetry of the source and moderator combination turns the helicity into a nonzero net positron polarization with spins parallel to the average positron velocity along the beam axis [4]. The beam polarization is defined as $p \equiv\left(N_{\uparrow}-N_{\downarrow}\right) /\left(N_{\uparrow}+N_{\downarrow}\right)$, where $N_{\uparrow(\downarrow)}$ is the number of positrons with spin parallel (antiparallel) to the beam axis. Backscattering in the source, absorption in the source material, and depolarization in slowing down will all affect the actual beam polarization, but typically one would expect $p \approx 0.5 h=35 \%$ for a beam derived from a ${ }^{22} \mathrm{Na}$ source [5]. We note that using a $\mathrm{MgO}$ moderator and a ${ }^{58} \mathrm{Co}$ source (that has virtually the same helicity as ${ }^{22} \mathrm{Na}$ ) a beam polarization of around $22 \%$ was observed [4].

Spin-polarized positron sources and beams have been used for $50 \mathrm{yr}$ to measure electron spin momentum distributions in ferromagnets [6], observe surface magnetism [7], search for the origin of optical activity in biological molecules [8], and study fundamental interactions $[9,10]$. With the advent of high density positron pulse techniques, the possibility of producing a Bose-Einstein condensate (BEC) of spin-polarized Ps atoms [11] has made it imperative to demonstrate some means of forming a fully spinpolarized collection of Ps. Here we present experimental proof that efficient preferential destruction of the minority spin atoms can occur in a high density collection of Ps, leaving us with a fully spin-polarized Ps gas. A concomitant beneficial result is that we have found a new way to measure the polarization of a high density positron pulse.

Our experiment examines Ps atoms interacting with each other in porous silica films [12]. Ps formed in the bulk material is emitted into the pores, and then hops amongst the interconnected voids, annihilating at a rate that typically depends on their size [13] and nature [14]. If the pores communicate with the sample surface the Ps may diffuse out of the film entirely, eventually annihilating in vacuum. If the electrons and positrons from which the Ps is formed are unpolarized, then the four lowest energy levels, a singlet and three triplet states, will each be created with equal likelihood. If some degree of polarization does exist then there will be a corresponding excess of (say) $m=1$ states, where $m$ refers to the projection of the spin quantum number along the beam axis. When oppositely polarized Ps atoms interact with each other they may engage in a variety of scattering processes [15]. The interactions apropos to this work are

$$
\begin{aligned}
& \mathrm{Ps}_{m=1}+\mathrm{Ps}_{m=-1} \rightarrow 2\left(\mathrm{Ps}_{m=0}+E_{\mathrm{hfs}}\right), \\
& \mathrm{Ps}_{m=1}+\mathrm{Ps}_{m=-1} \rightarrow \mathrm{Ps}_{2}+E_{B} .
\end{aligned}
$$

Here $E_{\mathrm{hfs}}$ is the hyperfine energy difference between the $|m|=1$ and $m=0$ states in the magnetic field [16] and $E_{B}=0.435 \mathrm{eV}$ is the $\mathrm{Ps}_{2}$ binding energy [17]. Any of these quenching processes will convert two long-lived (142 ns) triplet atoms into states that are quickly removed from the system, since $\mathrm{Ps}_{2}$ has a lifetime of $\sim 0.25 \mathrm{~ns}$, and both the singlet and triplet $m=0$ states have lifetimes of $<1.4 \mathrm{~ns}$ in the $2.3 \mathrm{~T}$ field of our target region. The reason for the latter is that in a strong magnetic field these states are mixed and, due to its short intrinsic lifetime (125 ps), even a small admixture of the singlet state results in a dramatically reduced triplet lifetime. This process, known as magnetic quenching, is illustrated in Fig. 1, which shows the delayed fraction $f_{d}$ as a function of the axial magnetic field at the target. The parameter $f_{d}$ is obtained from single shot lifetime spectra [18] recorded using a $\mathrm{PbWO}_{4}$ scintillator coupled to a fast photomultiplier tube [19] and is defined as the fraction of such spectra in the interval 50$300 \mathrm{~ns}$ [20]; the amount of long-lived positronium produced is approximately proportional to $f_{d}$ [12]. 


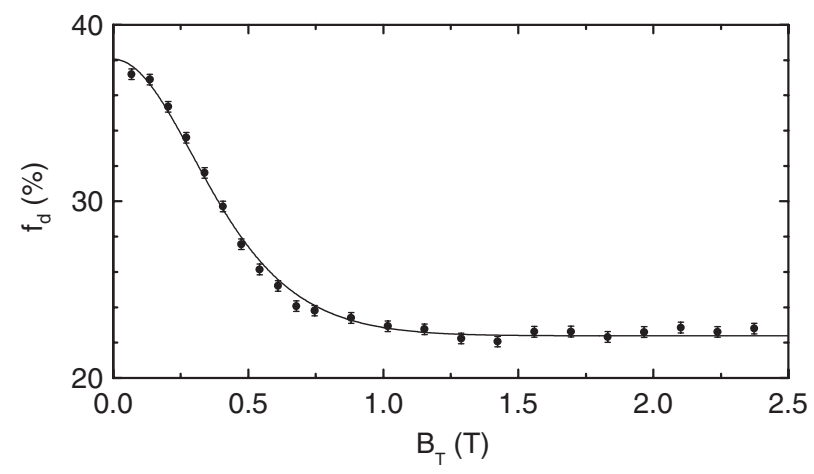

FIG. 1. The delayed fraction measured at low beam density and impact energy for different target magnetic fields, $B_{T}$. The magnetic quenching curve was fitted to the standard theory [16] assuming zero polarization and a vacuum decay rate of $130 \mathrm{~ns}^{-1}$.

The experiments were carried out using a positron accumulation system described elsewhere [21]. Positrons emitted from a ${ }^{22} \mathrm{Na}$ source and neon moderator are captured in a two stage Surko trap [22] and then transferred to a UHV accumulator, where a non-neutral plasma [23] is formed. These are compressed using the rotating wall (RW) technique [24] in the strong drive regime [25,26], which allows for precise control of the plasma density via the RW drive frequency, $f_{\mathrm{RW}} \cdot \mathrm{SF}_{6}$ cooling gas is used in the accumulator at a pressure of $1 \times 10^{-7}$ Torr. Positrons are accumulated for a few hundred seconds and compressed via the RW drive before the beam is implanted into the target with an approximately Gaussian areal distribution, with central density $n_{2 D}=$ $N f_{\mathrm{RW}}\left(4 \pi \varepsilon_{0} / e\right) B_{T} L_{p}$, where $L_{P}=5 \mathrm{~cm}$ is the plasma length and $B_{T}$ is the magnetic field at the target.

Positrons in the accumulator, where the axial magnetic field is $0.07 \mathrm{~T}$, are compressed to central areal densities up to $4 \times 10^{9} \mathrm{~cm}^{-2}$, which increases to more than $1 \times$ $10^{11} \mathrm{~cm}^{-2}$ in the $2.3 \mathrm{~T}$ magnetic field at the target. The positron pulses are compressed to $\sim 1 \mathrm{~ns}$ (FWHM) using an electrostatic buncher and may be accelerated into the target material with mean impact energies $K$ ranging from 2 to $8 \mathrm{keV}$. The positrons stop in the target with a mean implantation depth $\bar{z}$ related to the positron implantation energy by $\bar{z}=A \rho^{-1} K^{\nu}$ [27], where $K$ is in $\mathrm{keV}, \nu \approx 1.7, A$ is a sample dependent constant $\left(2.8 \mu \mathrm{g} / \mathrm{cm}^{2}\right)$ [28], and $\rho$ is the average target material density $\left(1.35 \mathrm{~g} / \mathrm{cm}^{3}\right)$. The mean time Ps spends in the sample before it is able to diffuse out to the vacuum depends on the implantation depth and in the present case $(K=6.2 \mathrm{keV})$ is around $\sim 0.5 \gamma^{-1}=25 \mathrm{~ns}$, where $\gamma^{-1} \approx 50 \mathrm{~ns}$ is the Ps lifetime in the pores when no Ps escapes [20].

The Ps formation target used in this work was an uncapped $600 \mathrm{~nm}$ thick porous $(\sim 50 \%)$ silica film deposited on a Si wafer substrate. The pores were considered to be randomly aligned, (insofar as they yielded no x-ray diffraction patterns) and had a diameter of $\sim 2.7 \mathrm{~nm}$. Ps formation and cooling in exactly similar films is described in detail in [20].

Figure 2 shows the delayed fraction and the positron beam density measured for different RW drive frequencies with $K=6.2 \mathrm{keV}$. The loss of compression at some frequencies (most prominently at $\sim 9 \mathrm{MHz}$ ) is due to zero frequency modes (ZFMs) that cause plasma heating (see $[25,26]$ for details). The reduction in the delayed fraction with increasing density is initially approximately linear, as has been previously observed [12], but then begins to saturate to a nonzero limiting value; we attribute this to the production of an almost polarized Ps ensemble, as we shall now explain.

Since the electrons in the target material are unpolarized, the initial polarization of Ps atoms is expected to be the same as that of the positrons. This means that a few nanoseconds after the beam is implanted, when the $m=0$ atoms have mostly decayed, we will be left with unequal numbers $n_{\uparrow(\downarrow)}$ of Ps atoms with $m= \pm 1$. The decay rates of these two populations are given by the sum of the low density decay rate for $|m|=1$ Ps atoms in the pores plus a nonlinear decay rate due to Ps-Ps collisions, which could lead to either of the channels of Eq. (1). The rate of nonlinear decay is proportional to the density of oppositely polarized $|m|=1$ Ps atoms, leading to the rate equations for the two populations:

$$
d n_{\uparrow(\downarrow)} / d t=-\gamma n_{\uparrow(\downarrow)}-\gamma \beta n_{\uparrow} n_{\downarrow} .
$$

Here $\beta$ is an effective interaction volume, confined within which two oppositely polarized Ps atoms would quench [see Eq. (1)] at a rate equal to $\gamma$. Defining two amplitudes $a_{\uparrow(\downarrow)}=\left(n_{\uparrow \downarrow l)} / n_{0}\right) \exp \{\gamma t\}$, with $n_{0}=n_{\uparrow}(0)+n_{\downarrow}(0)$, allows us to rewrite Eq. (2) as

$$
d a_{\uparrow(\downarrow)} / d t=-\gamma \beta n_{0} a_{\uparrow} a_{\downarrow} \exp \{-\gamma t\} .
$$

Note that these amplitudes would maintain their initial values $a_{\uparrow(\downarrow)}^{0}$ in the absence of Ps-Ps interactions, and that

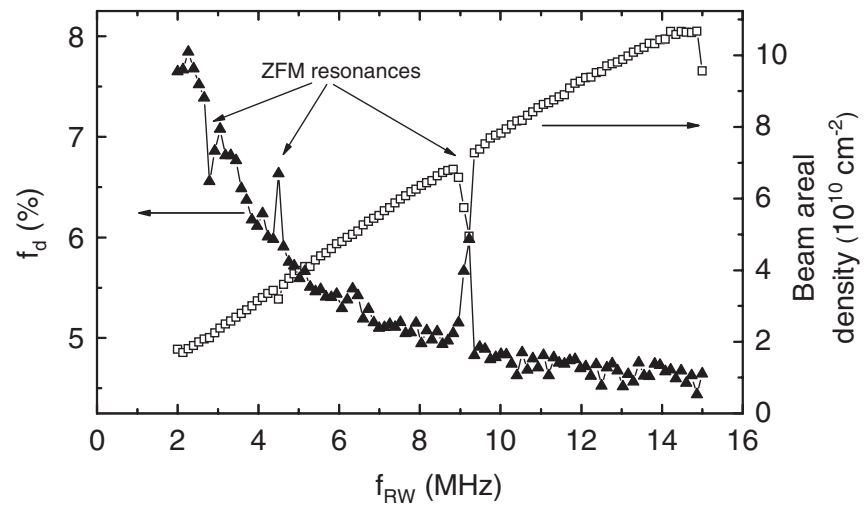

FIG. 2. Delayed fraction $(\boldsymbol{\Delta})$ and beam areal density on target ( $\square$ ) as a function of the RW compression frequency for positron implantation energy $K=6.2 \mathrm{keV}$ and magnetic field $B_{T}=$ $2.3 \mathrm{~T}$. 
any changes in the amplitudes will be equal and opposite (i.e., $\Delta a_{\uparrow}=-\Delta a_{\downarrow}$ ). Therefore, if we achieve a limit in which the minority spin atoms have decayed to zero by destroying an equal number of majority spin atoms, we will have for the remaining population $a_{\uparrow}=a_{\uparrow}^{0}-a_{\uparrow}^{0}=p_{0}$.

The decay rate of Ps in the sample is given by Eq. (2). After an average time $\bar{t}$, the Ps will have diffused out of the sample into the vacuum, where it will annihilate at a rate $\gamma_{0}$ that is slightly less than the vacuum decay rate because of wall collisions. At a time $t_{1}=50 \mathrm{~ns}>\bar{t}$ that marks the beginning of our delayed fraction interval $\left(t_{1}, t_{2}\right)$, the number of $|m|=1$ Ps atoms in vacuum will be $n(\beta, \vec{t}) \times$ $\exp \left\{-\gamma_{0}\left(t_{1}-\bar{t}\right)\right\}$, yielding a delayed fraction equal to a constant times the density at time $t_{1} \approx 50 \mathrm{~ns}$,

$$
f_{d}\left(\beta, t_{1}\right)=\text { const } \times n(\beta, \vec{t}) n_{0}^{-1} e^{-\gamma_{0}\left(t_{1}-\bar{t}\right)}\left(1-e^{-\gamma_{0}\left(t_{2}-t_{1}\right)}\right) .
$$

Normalizing our measured delayed fractions to the low density limit defines an empirical quenching parameter $Q=f_{d} / f_{d}^{0}$, which we see is theoretically identical to the sum of the two amplitudes $a=a_{\uparrow}+a_{\downarrow}$ :

$$
Q=f_{d}(\beta, \bar{t}) / f_{d}(0, \bar{t})=n(\beta, \bar{t}) n_{0}^{-1} e^{\gamma \bar{t}}=a(\beta, \bar{t}) .
$$

The latter obeys the equation

$$
d a / d t=-\frac{1}{2} \gamma \beta n_{0}\left(a^{2}-p_{0}^{2}\right) \exp \{-\gamma t\},
$$

which has the solution

$$
a(\zeta)=p_{0}\left[1+p_{0} \tanh \left\{\frac{1}{2} p_{0} \zeta\right\}\right] /\left[p_{0}+\tanh \left\{\frac{1}{2} p_{0} \zeta\right\}\right],
$$

where $\zeta \equiv \beta n_{0}[1-\exp \{-\gamma \vec{t}\}]$. The limiting values of the quenching parameter are thus $Q(0)=1$ (by definition) and $Q(\infty)=p_{0}$. Figure 3 shows the normalized delayed fraction data $Q$ and a fit to the measurements using Eq. (7), from which we obtain an initial beam polarization $p_{0}=$

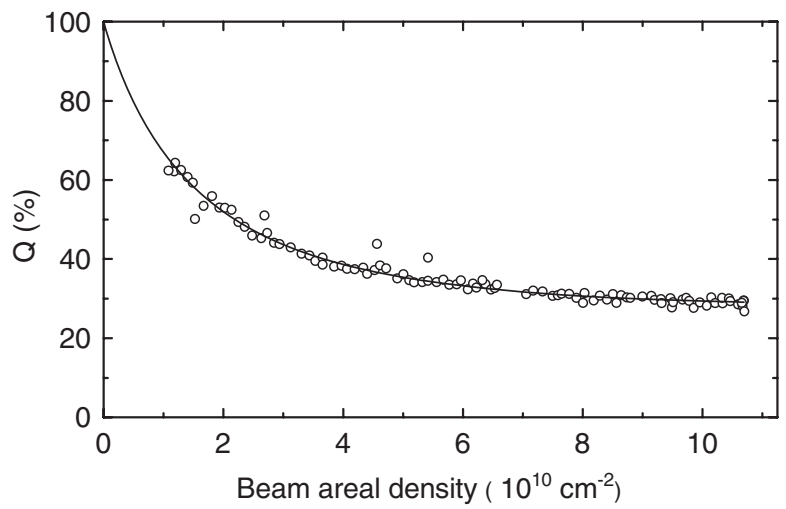

FIG. 3. Delayed fraction data of Fig. 2 normalized and given in terms of the beam areal density $n_{2 D}$. The solid line is a fit using the function defined in Eq. (7) including a normalization coefficient $(0.091 \pm 0.004)$, the polarization $p_{0}$, and a constant $\alpha$ as free parameters, where $\alpha \equiv \zeta / n_{2 D}$. Data points obviously associated with the ZFM resonances (see Fig. 2) have been excluded from the fit.
$28 \pm 1 \%$, a final Ps polarization $p_{m=1}=96 \pm 4 \%$ and a (maximum) value of the parameter $\zeta=12 \pm 1$ [29]. The latter implies that $\beta$ is of the order of $10^{-14} \mathrm{~cm}^{3}$.

The silica film used in this work [30] is not thought to support a Ps surface state [31] and, due to the random pore distribution, is also expected to suppress interactions in which oppositely polarized triplet atoms scatter into $m=0$ states [32], implying that the observed effect is probably due to the formation of molecular positronium [33] from free particle cavity Ps states. This process, which would usually be suppressed due to momentum conservation, is possible by virtue of the significant overlap of the wave function of thermalized Ps with the walls of the $\sim 2.7 \mathrm{~nm}$ diameter pores [20]. It is possible that there might be an unexpected Ps surface state (with a high activation energy), or that there may be enough short-range order in the pore structure to allow for scattering into singlet states without the film producing an $\mathrm{x}$-ray diffraction pattern. Thus, we cannot definitively say which process of Eq. (1) we have observed, but the saturation of the quenching effect will occur with the same dependence on the positron polarization in either case. The surprisingly large value obtained for the effective interaction volume $\beta$ (equivalent to around $10^{6}$ pores) could indicate that some unknown property of the film structure leads to an enhanced rate of quenching, such as the existence of large pores where Ps atoms accumulate.

The measured value of $p_{0}$ is close to what we would expect for the primary positron beam, which indicates that the capture and storage of positrons in a buffer gas trap does not lead to any significant depolarization, despite storage in a magnetic field for some $10^{12}$ cyclotron orbits. Although we would not expect the electromagnetic fields of a perfectly aligned Penning trap to depolarize positrons contained therein, there are mechanisms associated with inhomogeneous fields (as might be generated by trap imperfections), plasma waves, or collisions with gas molecules that could [34]. It is therefore valuable to experimentally demonstrate that the initial positron spin states are essentially maintained in Surko-type accumulators. In future work it would be useful to measure $p_{0}$ for the incident dc beam and the trapped beam separately, so as to accurately determine the extent of any trap induced depolarization that might occur.

The production of spin-polarized Ps is critical for any experimental attempt to create a Ps BEC [11] since, at the high densities required for a phase transition to occur at achievable temperatures, an unpolarized collection of Ps would undergo total spin conversion and be quickly removed from the system. The present methodology results in a Ps density thought to be at least 2 orders of magnitude too low to achieve Bose-Einstein condensation of Ps [33]. A number of experimental improvements, including increasing the primary beam polarization, remoderating the beam [11], using multiple coaxial positron traps, target 
structures that enhance the collection of Ps into single large cavities, and Ps laser cooling [35], may change this. However, no such improvements to the experimental methods would be useful were it not the case that the initial positron polarization is preserved in the accumulation process, and the production of a spin-polarized Ps gas therefore represents an important development towards producing a Ps BEC.

To summarize, we have observed the saturation of density dependent quenching due to Ps-Ps interactions. We attribute this to the removal of the minority spin component, resulting in a fully polarized Ps ensemble, as required for the production of a Ps BEC. Our data show conclusively that there is no significant loss of polarization when positrons are accumulated in a Surko trap, which had not previously been demonstrated. Although we cannot definitively identify the quenching mechanism, the known properties of the sample point towards the formation of molecular positronium, which could mean that this methodology will be suitable for laser spectroscopy of excited molecular Ps states [17].

We are grateful to R. G. Greaves for discussions and P. Crivelli, L. Liszkay, P. Perez, and co-workers for providing porous silica samples. This work was supported in part by the National Science Foundation under Grant No. PHY 0900919 and by the U.S. Air Force Research Laboratory.

[1] T. D. Lee and C. N. Yang, Phys. Rev. 104, 254 (1956).

[2] J.D. Jackson, S. B. Treiman, and H. W. Wyld, Jr., Phys. Rev. 106, 517 (1957).

[3] K. F. Canter, P. G. Coleman, T. C. Griffith, and G. R. Heyland, J. Phys. B 5, L167 (1972).

[4] P. W. Zitzewitz, J.C. Van House, A. Rich, and D. W. Gidley, Phys. Rev. Lett. 43, 1281 (1979).

[5] Positrons produced by a ${ }^{22} \mathrm{Na}$ source have an average helicity of about $70 \%$. See, for example, P. G. Coleman and N. R. Potter, Appl. Surf. Sci. 255, 101 (2008).

[6] S. Berko and J. Zuckermann, Phys. Rev. Lett. 13, 339 (1964).

[7] D. W. Gidley, A. R. Koymen, and T. W. Capehart, Phys. Rev. Lett. 49, 1779 (1982).

[8] A. Rich and J. Van House, Orig. Life Evol. Biosph. 16, 423 (1986).

[9] A. Rich and H. R. Crane, Phys. Rev. Lett. 17, 271 (1966).

[10] M. Skalsey, T. A. Girard, D. Newman, and A. Rich, Phys. Rev. Lett. 49, 708 (1982).

[11] P. M. Platzman and A. P. Mills, Jr., Phys. Rev. B 49, 454 (1994).
[12] D. B. Cassidy, S. H. M. Deng, R. G. Greaves, T. Maruo, N. Nishiyama, J. B. Snyder, H. K. M. Tanaka, and A. P. Mills, Jr., Phys. Rev. Lett. 95, 195006 (2005).

[13] D. W. Gidley, W. E. Frieze, T. L. Dull, A. F. Yee, E. T. Ryan, and H.-M. Ho, Phys. Rev. B 60, R5157 (1999).

[14] For example, C. He et al., Phys. Rev. B 75, 195404 (2007).

[15] I. A. Ivanov, J. Mitroy, and K. Varga, Phys. Rev. Lett. 87, 063201 (2001); I. A. Ivanov, J. Mitroy, and K. Varga, Phys. Rev. A 65, 022704 (2002).

[16] A. Rich, Rev. Mod. Phys. 53, 127 (1981).

[17] For example, J. Usukura, K. Varga, and Y. Suzuki, Phys. Rev. A 58, 1918 (1998).

[18] D. B. Cassidy, S. H. M. Deng, H. K. M. Tanaka, and A. P. Mills, Jr., Appl. Phys. Lett. 88, 194105 (2006).

[19] D. B. Cassidy and A. P. Mills, Jr., Nucl. Instrum. Methods Phys. Res., Sect. A 580, 1338 (2007).

[20] D. B. Cassidy, P. Crivelli, T. H. Hisakado, L. Liszkay, V. E. Meligne, P. Perez, H. W. K. Tom, and A. P. Mills, Jr., Phys. Rev. A 81, 012715 (2010).

[21] D. B. Cassidy, S. H. M. Deng, R. G. Greaves, and A.P. Mills, Jr., Rev. Sci. Instrum. 77, 073106 (2006).

[22] R. G. Greaves and C.M. Surko, Phys. Plasmas 4, 1528 (1997).

[23] R.C. Davidson, Physics of Non-Neutral Plasmas (Addison-Wesley, Redwood City, 1990).

[24] F. Anderegg, E. M. Hollmann, and C.F. Driscoll, Phys. Rev. Lett. 81, 4875 (1998); R. G. Greaves and C.M. Surko, Phys. Rev. Lett. 85, 1883 (2000).

[25] J. R. Danielson and C. M. Surko, Phys. Rev. Lett. 94, 035001 (2005).

[26] D. B. Cassidy, R. G. Greaves, V.E. Meligne, and A.P. Mills, Jr., Appl. Phys. Lett. 96, 101502 (2010).

[27] A. P. Mills and R. J. Wilson, Phys. Rev. A 26, 490 (1982).

[28] J. Algers, P. Sperr, W. Egger, G. Kögel, and F. H.J. Maurer, Phys. Rev. B 67, 125404 (2003).

[29] The quoted error estimates are double the statistical errors to allow for possible systematic effects.

[30] We note that prolonged irradiation with the high density positron beam creates some radiation damage in the sample. See also D. B. Cassidy and A. P. Mills, Jr., Nucl. Instrum. Methods Phys. Res., Sect. B 262, 59 (2007).

[31] P. Crivelli, U. Gendotti, A. Rubbia, C. Corbel, L. Liszkay, and P. Perez (to be published).

[32] Outgoing states must be able to accommodate the hyperfine energy difference between the $|m|=1$ and $m=0$ states, which means that this process may be suppressed if a continuum of cavity states does not exist, as would be the case for random pores. D. B. Cassidy and A. P. Mills, Jr., Phys. Rev. Lett. 100, 013401 (2008).

[33] D. B. Cassidy and A. P. Mills, Jr., Nature (London) 449, 195 (2007).

[34] S.-J. Chen and D.H.E. Dubin, Phys. Fluids B 5, 691 (1993).

[35] E.P. Liang and C.D. Dermer, Opt. Commun. 65, 419 (1988). 